\title{
MRI-Guided Breast Biopsy-Histopathological Verification of Suspicious Breast Lesions Visible on MRI Only
}

\author{
Heinze $S^{1}$, Rudnicki $W^{2}$, Popiela $T^{3}$ and Luczynska \\ $\mathbf{E}^{4 *}$ \\ ${ }^{1}$ Maria Sklodowska-Curie National Research Institute of \\ Oncology in Krakow, Poland \\ 2Jagiellonian University Medical College, Poland \\ ${ }^{3}$ Department of Radiology, Jagiellonian University \\ Medical College, Poland \\ ${ }^{4}$ University of Rzeszow, Faculty of Medicine, Rzeszow, \\ Poland \\ *Corresponding author: Elzbieta Luczynska, \\ Univeristy of Rzeszow, Faculty of Medicine, Rzeszow, \\ Poland
}

Received: October 26, 2020; Accepted: November 26, 2020; Published: December 03, 2020

DOI: https://doi.org/10.26420/austinjradiol.2020.1117

\begin{abstract}
Background: Breast cancer is currently the most frequently diagnosed cancer in women. While the range of modalities enabling suspicious lesions detection is wide, MRI remains the most sensitive one. Therefore, the number of methods verifying the lesions seen only on MRI images grows. The aim of this study is to check the usefulness of MRI guided breast biopsy in clinical use.

Methods: The study involved 120 patients who underwent diagnostic MRI before the biopsy that revealed suspicious lesions (BI-RADS 4 and 5). Those lesions had not been seen on initial ultrasonography or mammography. In each case, a marker was placed in the biopsy site and histopathological examination of the obtained samples was performed.
\end{abstract}

Results: The study revealed benign lesions in 86 patients $(71.7 \%)$. The remaining 34 lesions $(28.3 \%)$ were determined as malignant, including 19 noninfiltrating $(15.8 \%)$ and 15 infiltrating lesions $(12.5 \%)$. Study showed correlation between kinetic curve type and lesion malignancy. Breast type, BPE and enhancement type did not have impact on the histopathology result.

Conclusion: Breast MRI-guided biopsy is a reliable way to verify lesions not visible on any other diagnostic imaging methods and therefore should be developed.

Keywords: Breast cancer; Biopsy; MRI

\section{Introduction}

Breast imaging is a difficult and unique examination, due to wide range of different imaging methods. Mammography is based on $\mathrm{X}$-ray radiation, whereas ultrasonography does not include radiation. Another, more expensive method-MRI (Magnetic Resonance Imaging) is performed after contrast agent administration. These methods are complementary to each other. According to European Society of Breast Imaging recommendations mammography is a basic diagnostic method of clinically asymptomatic breast cancer [1]. However, it is worth remembering, that sensitivity and specificity of mammography is limited, mostly by dense breast anatomy $[2,3]$. Therefore, other modalities are applied, such as ultrasonography and MRI $[4,5]$. In breast cancer diagnostics a radiologist, being a member of a multidisciplinary team is considered to be directly and personally responsible for the patient. Ability to diagnose a suspicious breast lesion is highly dependent on radiologist's knowledge and intuition [6].

Contrast-enhanced breast MRI is the most sensitive technique for breast cancer detection and is commonly used for breast cancer screening in high-risk patients. This method has high sensitivity as high as $100 \%$ for breast cancer, but its specificity is lower (37$78 \%$ ), so in some cases biopsy is required to establish a diagnosis $[7,8]$. High sensitivity allows visualizing lesions that were not detected by other imaging modalities. In patients with suspicious lesions not identified in retrospective ultrasonography and occult in mammography histological verification with MRI-guided breast biopsy is recommended [7,9-12].
MRI-guided breast biopsy is an expensive procedure that requires dedicated equipment and experienced staff, which makes careful selection of suspicious cases highly important.

The aim of this study was to correlate MRI-guided breast biopsy results to initial MRI findings.

\section{Materials and Methods}

The study initially involved 164 patients who had lesions visible only on breast MRI. In 35 patients, MRI performed prior to biopsy did not confirm the lesion presence, while 9 patients had lesions unavailable for biopsy under MRI guidance. As a result, the material included 120 patients who underwent vacuum assisted core needle biopsy under MRI guidance.

Benign lesions were found in 86 patients (71.7\%), including 38 (31.7\% of the whole) lesions type B3. The remaining 34 lesions (28.3\%) were determined as malignant, including 19 non-infiltrating (15.8\%) and 15 infiltrating lesions (12.5\%).

The average age of the patients (median) was 50 years (ranges from 29 to 82 ).

This study was performed in compliance with the Declaration of Helsinki and it received the approval of the Ethical Committee at the Regional Medical Chamber (acceptance No. OIL/KBL/17/2018).

\section{MRI protocol and breast biopsy equipment}

MRI examination was performed in prone position on a $1.5 \mathrm{~T}$ Siemens Avant unit with a dedicated, RF phased array breast coil 
(Noras MR products), which had open access to the lateral or medial part of the breast depending on the location of the suspicious lesion. MR-compatible localization system was applied, including grid/post\&pillar system, marker cube fulfilled with vitamin $\mathrm{A}+\mathrm{E}$ (improving visibility on MR images) and introducer biopsy pack (with dedicated biopsy needle).

The breast biopsy MRI protocol differs from the diagnostic one it is based on repeated several times $3 \mathrm{D}$ T1 weighted sequence without fat saturation. It is accurate to visualize enhancing lesions after intravenous contrast injection $(0.1 \mathrm{mmol} / \mathrm{kg}$ gadolinium based agent) on subtraction images; position of the biopsy chamber inserted into a patient before tissue sampling and, after the procedure-to visualize the applied marker.

Indications and contraindications for MRI-guided breast biopsy are the same as for conventional MRI examination and include having implanted pacemakers or metal stents; claustrophobia and allergic reaction to gadolinium based contrast agents or local anesthesia [13]

Prior to the procedure, the patients were asked about the use of medications such as aspirin, anticoagulants or other agents known to impact bleeding time. All those factors were taken under consideration before the examination and biopsy.

Before the procedure, the diagnostic MRI was reviewed for the best patient positioning and approach planning. Depending on suspicious lesion location, the proper needle ( 115 or $145 \mathrm{~mm}$; $8 \mathrm{G}$ or $11 \mathrm{G})$ was chosen. Patients were examined in the prone position with breast compressed with grid plates to avoid body movements and enable tissue sampling.

\section{Evaluation}

Biopsy was performed by a radiologist with 20-year experience in breast cancer diagnostics, including 10-year experience in performing core needle biopsy under US or MG guidance and 10year in MRI assessment. Two radiologists evaluated the results of MRI examination before biopsy: one of them with 10-year experience, while the second with 8-year experience in MRI assessment. Both of them performed retrospective ultrasound examinations under MRI images guidance. In case of negative retrospective ultrasound results, the decision to perform MRI-guided biopsy was made.

The patients were referred to biopsy under MRI guidance following MRI and retrospective breast ultrasound. If no focal lesions were found on ultrasound in projection of the lesion previously described on mammography examination, biopsy under MRI guidance was performed. MRI was scheduled during follicular phase, i.e., between 7-14 day of the patient's menstrual cycle and so was the biopsy under MRI guidance [14,15].

Breast anatomy was evaluated in all patients and determined as fatty, glandular or mixed. Parenchymal enhancement was assessed and divided into minimal, mild, moderate and marked in concordance with the BI-RADS recommendations.

Lesions found on MRI examination were classified as mass or non-mass-like enhancement lesions according to BI-RADS (Breast Imaging-Reporting and Data System) classification. Non-mass-like enhancement types were further divided into focal, linear, segmental, regional, multifocal and diffuse enhancement. Enhancement kinetic curves were evaluated for all enhancing lesions. The evaluation was also based on BI RADS classification and the curves were divided into three types: persistent, plateau and washout.

\section{Procedure}

The biopsy was performed with Mammotome (Leica) system by the radiologist interpreting the initial examination. Minimal number of samples obtained from every patient was 12 , which means 1 sample for each part of the breast using the o'clock position. After the procedure, a marker was placed in the biopsy site Figure 1.

For lesions localized too close to the chest wall or to the skin surface, a marker clip was inserted under MRI guidance into the suspicious area and then a wire localization procedure under mammography or US could be performed prior to tumorectomy, instead of biopsy.

\section{Histopathology}

Further steps included placing the samples in buffered formalin solution before sending them to the Pathology Department. Standard hematoxylin and eosin staining was performed and the specimens obtained were evaluated by a pathologist with at least 5-year experience in breast cancer diagnostics. Every single specimen was an object of evaluation. If breast cancer was determined, ER, PR and HER status, as well as Ki67 index were additionally identified to plan the patient management effectively.

\section{Results}

\section{Relation between biopsy under MRI guidance results and patient's age}

The lesion grade differs significantly depending on age the most malignant lesions are more frequent in older patients. The differences between age medians in patients with benign lesions, B3 and noninfiltrating are statistically insignificant. However, the average age of patients with benign lesions and lesions type B3 is statistically significantly smaller $(p<0.02)$ than the average age of the patients with malignant infiltrating lesions Figure 2.

\section{Breast anatomy type}

Three types of breast anatomy were determined within the examined group of patients: glandular (17-14.2\%), fatty 14 (11.7\%), mixed (89-74.1\%).

Because of presented above results it is not possible to determine if a certain anatomy type is more characteristic for malignant lesions than benign. Frequency of anatomy type incidence is similar

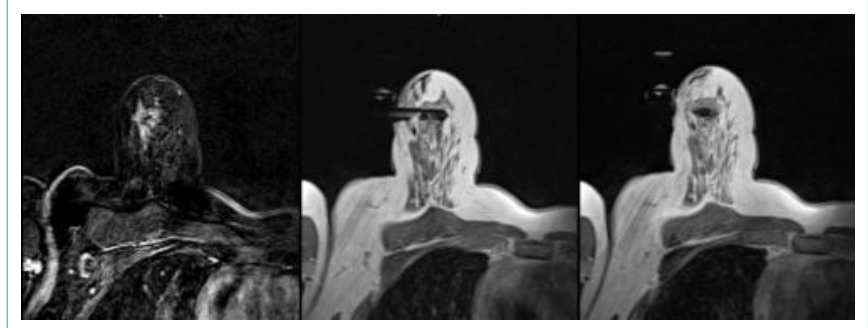

Figure 1: Images acquired during the procedure. From the left T1WI post contrast subtraction to localize the lesion, T1WI post contrast with needle inserted to check the position of cutting chamber, T1WI post contrast after marker placing to verify its position. 


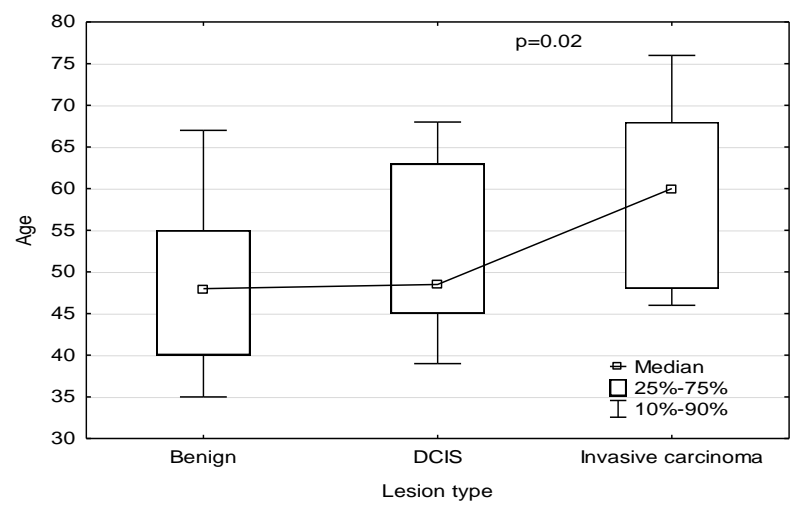

Figure 2: Relation between patients age and lesion grade.

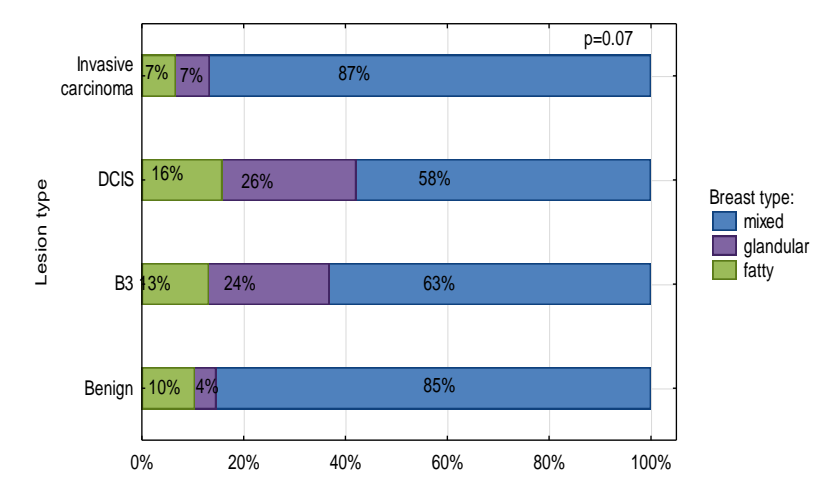

Figure 3: Relation between lesion grade and breast type.

for benign and malignant lesions. When lesions are divided into malignant non-infiltrating, infiltrating and benign with $\mathrm{B} 3$ type, breast anatomy is also insignificant ( $\mathrm{p}=0.36$ ). With such a division of lesions it can be observed that glandular breast type is the most frequent in case of non-infiltrating lesions (26\%), being more frequent than in case of benign lesions with B3 or infiltrating (13\%, 7\%), however this difference is statistically non-significant ( $p=0.17,0.10)$. Mixed breast type is less common in case of non-infiltrating lesions (58\%) in comparison with infiltrating lesions (87\%) and benign with B3 (76\%). These differences are statistically insignificant, $\mathrm{p}=0.054$ and 0.014 , respectively. If division that is more detailed is applied (lesions B3 separated), it can be noticed that frequency of incidence of individual breast anatomy types is similar for non-infiltrating lesions (in situ) and B3. Nevertheless, there is no significant relation between lesion type (infiltrating, non-infiltrating, B3, benign) and breast anatomy type $(\mathrm{p}=0.07)$. Glandular breasts are more common in case of B3 and non-infiltrating lesions (26 and $24 \%$, respectively) than in case of benign lesions ( $4 \%)(\mathrm{p}=0.005$ and $\mathrm{p}=0.01$, respectively). In case of infiltrating lesions glandular breasts (7\%) are also rarer than in lesions B3 and non-infiltrating, but this relation is statistically insignificant $(\mathrm{p}=0.1)$. Breasts with mixed anatomy type are rarer in case of $\mathrm{B} 3$ and non-infiltrating lesions (63\% and $58 \%$, respectively) than in benign lesions $(85 \%)(\mathrm{p}=0.02)$. When it comes to infiltrating lesions, this relation is statistically insignificant $(\mathrm{p}=0.067$ and 0.054$)$ Figure 3.

\section{Types of enhancement}

Among the lesions, four types of enhancement were distinguished:

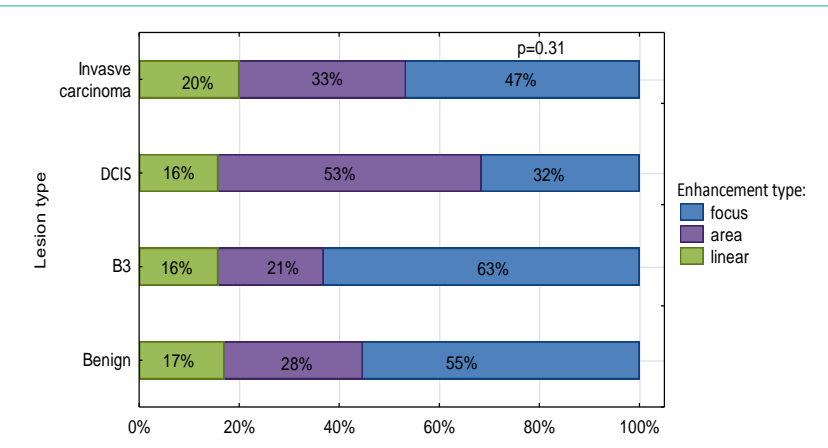

Figure 4: Relation between enhancement morphology and histological type of the lesions.

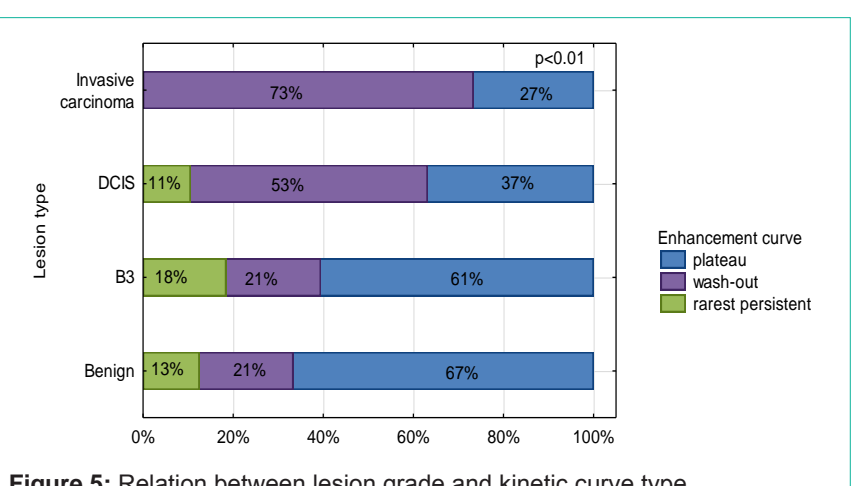

Figure 5: Relation between lesion grade and kinetic curve type.

mass (63-52.5\%) including ring enhancement (1-0.8\%) or regional (36-30.0\%) and linear (20-16.7\%) enhancement. Type of enhancement did not influence lesions division based on their type in other words, it did not depend on enhancement whether the lesion was malignant or not ( $\mathrm{p}>0.08)$. With more detailed analysis of lesions division based on enhancement type the results were as follows: linear type appeared with similar frequency in all types of lesions, but frequency of regional and mass enhancement was statistically significantly different in case of non-infiltrating lesions in comparison with B3 lesions. Regional type of enhancement was more common among non-infiltrating lesions (53\%) than among B3 lesions (21\%), p=0.02. Regional type of enhancement was rarer among non-infiltrating lesions (32\%) than among B3 lesions (63\%), p=0.02 Figure 4.

\section{Enhancement curve}

The most common type of enhancement curve found during MRI examination was plateau (66-55 \%), then washout (39-32.5\%) and the rarest - persistent (15-12.5\%). There was a strong significant relation between lesions division and curve type $(\mathrm{p}<0.01)$. Washout type curve was most frequent in case of malignant lesions, especially among infiltrating lesions (73\%). Plateau type was most common among benign lesions (13\%). It was noted, that the higher malignancy of the lesion, the higher washout type curve incidence and the rarer plateau incidence Figure 5.

\section{Lesion size}

The median lesion size determined on MRI examination was $16 \mathrm{~mm}$ ranging from 5 to $60 \mathrm{~mm}$. Malignancy of the lesion significantly depended on its size ( $\mathrm{p}=0.03$ ), thus basing on the lesion size it was possible to determine whether the lesion is malignant or benign. 


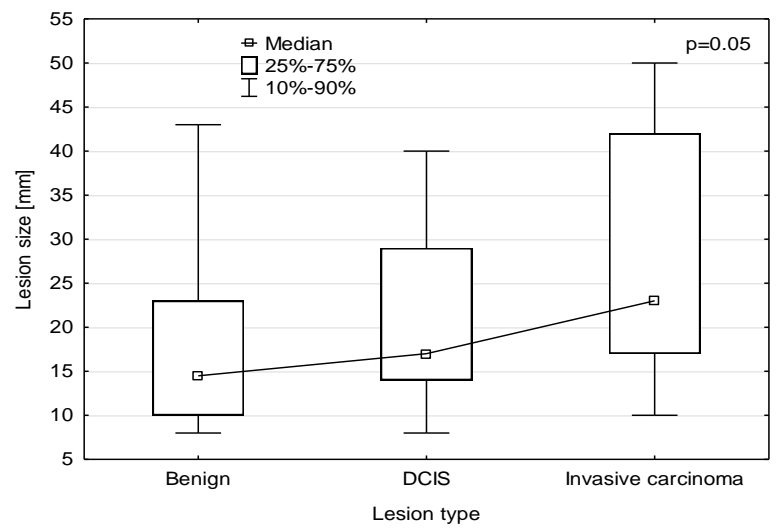

Figure 6: Relation between lesion size and its histologic grade.

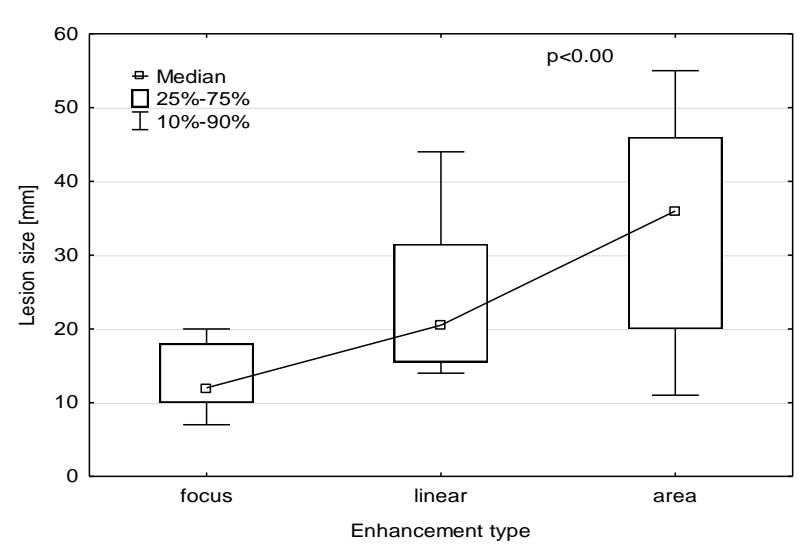

Figure 7: Relation between lesion size and its enhancement kinetic curve.

The lesion size was also correlated with histologic grade $(\mathrm{p}=0.05)$ the greater the lesion size, the higher the histologic grade Figure 6.

It was simultaneously observed that the lesion size was statistically significantly correlated with the enhancement type $(\mathrm{p}<0.001)$. The bigger lesion size was characteristic for the area enhancement type and the smaller one for the focus enhancement type Figure 7.

\section{Breast parenchymal enhancement}

The relation between parenchymal enhancement type and lesion type was determined as statistically insignificant, which meant that whether the lesion was malignant or benign did not affect the parenchymal enhancement type ( $\mathrm{p}>0.5)$.

The level of enhancement is not statistically dependent on lesion type, which means that basing on enhancement level it is not possible to determine whether the lesion is a cancer $(\mathrm{p}>0.06)$. However, it can be seen that enhancement level increases with malignancy of the lesion.

\section{Discussion}

Clinical application of breast MRI has recently increased due to its high sensitivity and unique ability to detect lesions not visible on other imaging modalities, including mammography and ultrasound. While the sensitivity is high, the specificity is not satisfactory, resulting from the fact that normal breast tissue or benign lesions show enhancement on MRI. Therefore, suspicious lesions found on MRI are subjected to mammography or ultrasound in order to confirm their presence and then imaging guided localization or biopsy procedure is performed [16]. However, using the three-dimensional information about lesion location from MRI requires knowledge and experience in both techniques, including the ability to process the data pertaining to the lesion position from one modality to another.

In cases when mammography or ultrasound is not sufficient for clear identification of the lesion, an MRI-guided biopsy is performed. Nevertheless, such a procedure generates higher costs and requires longer time, which make it less available. What is more, biopsy under MRI guidance is one of the most complicated techniques. Still, it remains a vital diagnostic tool, especially for patients with confirmed breast cancer and coexisting suspicious contrast enhancement foci in the same or contralateral breast not visible on other diagnostic imaging modalities. This method is safe for the patient and allows for an accurate breast lesion diagnosis [17]. An alternative to the biopsy under MRI guidance is marker placement under MRI guidance followed by an open surgical biopsy. Yet, this procedure is more invasive for the patient.

Our study involved 120 patients who underwent vacuum assisted core needle biopsy under MRI guidance. The study revealed benign lesions in 86 patients $(71.7 \%)$. The remaining 34 lesions $(28.3 \%)$ were determined as malignant, including 19 non-infiltrating (15.8\%) and 15 infiltrating lesions (12.5\%). The percentage of cancers verified by biopsy under MRI guidance is in concordance with ACR recommendations BI RADS 2013. According to the literature data, $50-70 \%$ of lesions were benign, whereas up to $30 \%$ of lesions were malignant [18].

Our study revealed that histologic grade of the lesions correlates with enhancement level on MRI. The lesion size significantly depended on its malignancy ( $\mathrm{p}=0.03$ ), thus basing on the lesion size it was possible to determine whether the lesion is malignant or benign. The lesion size was also correlated with histologic grade $(\mathrm{p}=0.05)$ the greater the lesion size, the higher the histologic grade.

Literature data also confirm lack of significant differences in upgrade rates between mass and non-mass enhancement or lesion size $[19,20]$. Such outcomes can result from the biologic heterogeneity of lesions or from the limitations of sampling during the biopsy under MRI guidance.

In our material, no statistical significance between MRI image characteristics and malignancy was obtained within the examined group of patients. It means, that before MRI guided biopsy all possible measures should be undertaken to ensure accurate evaluation of all characteristics of the lesion visible on examination and to perform retrospective ultrasound with high accuracy, using high-resolution equipment and MRI guidance in order to avoid unnecessary biopsy under MRI. Some lesions can be identified by comparing the characteristics with benign pathologies, which may be also helpful in avoiding biopsy [21].

Main limitations of the study are: small number of lesions resulting from highly advanced disease state, the examination cost and small number of patients referred to biopsy under MRI guidance.

Another limitation is the retrospective character of the study not 
allowing for full analysis of all data.

Long-term follow up of all the patients who underwent biopsy was not possible, which was also considered a limitation of the study. In Poland there are only three clinics where MRI guided biopsy is performed and our clinic is one of them. The patients arrive from distant parts of the country, which means that after the biopsy procedure some of them are subjected to surgery and stay under surveillance in their local clinics. For that reason, our data pertaining to those patients is not sufficient.

\section{Conclusion}

Breast MRI-guided biopsy is a reliable technique to verify lesions not visible on any other diagnostic imaging methods and therefore should be developed. Training new radiologist to perform the biopsy as well as making the procedure more known to other specialty doctors can positively influence breast cancer detection in early stages.

\section{Acknowledgement}

Authors would like to thank PhD Dorota Olszewska that kindly provided statistical advice for this manuscript.

\section{References}

1. Sardanelli F, Fallenberg EM, Clauser P, Trimboli RM, Camps-Herrero J, Helbich TH, et al. Mammography: an update of the EUSOBI recommendations on information for women. Insights Imaging. 2017; 8: 11-18.

2. Azzam H, Kamal RM, Hanafy MM, Youssef A, Hashem LMB. Comparative study between contrast-enhanced mammography, tomosynthesis, and breast ultrasound as complementary techniques to mammography in dense breast parenchyma. Egypt J Radiol Nucl Med. 2020; 51: 148.

3. Dibble EH, Singer TM, Jimoh N, Baird GL, Lourenco P. Dense breast ultrasound screening after digital mammography versus after digital breast tomosynthesis. Am J Roentgenol. 2019; 213: 1397-1402.

4. Kuhl CK, Schrading S, Leutner CC, Morakkabati SN, Wardelmann E Fimmers $\mathrm{R}$ et al. Mammography, breast ultrasound, and magnetic resonance imaging for surveillance of women at high familial risk for breast cancer. J Clin Oncol Off J Am Soc Clin Oncol. 2005; 23: 8469-8476.

5. Dromain C, Balleyguier C, Adler G, Garbay JR, Delaloge S. Contrastenhanced digital mammography. Eur J Radiol. 2009; 69: 34-42.

6. Kuhl CK: The changing world of breast cancer: A radiologist's perspective. Invest Radiol. 2015; 50: 615-628.

7. Han BK, Schnall MD, Orel SG, Rosen M. Outcome of MRI-guided breast biopsy. Am J Roentgenol. 2008.

8. Mahoney MC, Gatsonis C, Hanna L, DeMartini WB, Lehman C. Positive predictive value of BI-RADS MR imaging. Radiology. 2012; 264: 51-58.

9. Rauch GM, Dogan BE, Smith TB, Liu P, Yang WT. Outcome analysis of 9-gauge MRI-guided vacuum-assisted core needle breast biopsies. Am J Roentgenol. 2012; 198: 292-299.

10. Kamal RM, Helal MH, Mansour SM, Haggag MA, Nada OM, Farahat IG, et al. Can we apply the MRI BI-RADS lexicon morphology descriptors on contrastenhanced spectral mammography? Br J Radiol. 2016; 89: 20160157.

11. Papalouka V, Kilburn-Toppin F, Gaskarth M, Gilbert F. MRI-guided breast biopsy: a review of technique, indications, and radiological-pathological correlations. Clin Radiol. 2018; 73: 908.e17-908.e25.

12. Maglione KD, Lee AY, Ray KM, Joe BN, Balassanian R. Radiologicpathologic correlation for benign results after MRI-guided breast biopsy. Am J Roentgenol. 2017; 209: 442-453.

13. Ghadimi M, Sapra A. Magnetic Resonance Imaging (MRI), Contraindications. 2020.

14. Ellis RL. Optimal timing of breast MRI examinations for premenopausal women who do not have a normal menstrual cycle. Ajr Am J Roentgenol. 2009; 193: 1738-1740.

15. Liao GJ, Henze Bancroft LC, Strigel RM, Chitalia RD, Kontos D, Moy L, et al. Background parenchymal enhancement on breast MRI: A comprehensive review. J Magn Reson Imaging. 2020; 51: 43-61.

16. Gao $\mathrm{P}$, Kong $\mathrm{X}$, Song $\mathrm{Y}$, Fang $\mathrm{Y}$, Ouyang $\mathrm{H}$, et al. Recent Progress for the Techniques of MRI-Guided Breast Interventions and their applications on Surgical Strategy. J Cancer. 2020; 11: 4671-4682.

17. Hefler L, Casselman J, Amaya B. Follow-up of breast lesions detected by MRI not biopsied due to absent enhancement of contrast medium. Eur Radiol. 2003; 13: 344-346.

18. Ana PL, Hanan K, Matthew S, Linda D. High-Risk Lesions at MRI-Guided Breast Biopsy: Frequency and Rate of Underestimation American Journal of Roentgenology. 2014; 203: 682-686.

19. Roberta MS, Peter RE, Wendy BD, Robert LG, Kimberly H. Frequency, Upgrade Rates, and Characteristics of High-Risk Lesions Initially Identified With Breast MRI. Allison, Sue Peacock, and Constance D. Lehman American Journal of Roentgenology. 2010; 195: 792-798.

20. Crystal P, Sadaf A, Bukhanov K, McCready D, O'Malley F, Helbich TH. Highrisk lesions diagnosed at MRI-guided vacuum-assisted breast biopsy: can underestimation be predicted? Eur Radiol. 2011; 21: 582-589.

21. Hayward JH, Ray KM, Wisner DJ, Joe BN. Follow-up outcomes after benign concordant MRI-guided breast biopsy. Clin Imaging. 2016; 40: 1034-1039. 